Ann. Abeille, I963, 6 (3), I85-200.

\title{
LES ERREURS D'ORIENTATION DES AREILLES (DÉRIVE) DANS LE RUCHER MODERNE
}

\author{
J. FRESNAYE \\ Station expérimentale d'Apiculture, \\ (ientre de Recherches agronomiques du Sud-Est, Montfai'et (Vaucluse)
}

SOMMAIRE

L'auteur compare l'importance des erreurs d'orientation des abeilles dans trois ruchers voisins composés de ruches semblables et pendant la même période de l'année.

Les résultats obtenus montrent que la dérive peut atteindre de très fortes proportions dans les ruchers dont le site ne comporte aucun repère discernable par les abeilles. Les différences de rendement provoquées par la dérive, sont très importantes et $20 \mathrm{p}$. 100 des ruches ne récoltent pas de miel du tout.

La dérive diminue beaucoup dès que quelques points de repère permettent aux butineuses de s'orienter, et disparaît presque totalement lorsque les repères sont suffisants.

I.a surface de couvain des ruches subissant une forte dérive se trouve réduite du fait de l'affaiblissement des populations.

Il semble également que la dérive puisse provoquer un pillage pouvant aller jusqu'à la destruction des colonies attaquées.

\section{IN'TRODUCTION}

On sait que chez 1'Abeille, le sens de l'orientation est particulièrement développé. De nombreux travaux ont été consacrés à son étude et von FRISCH (I958) a pu écrire à son sujet " qu'on est tenté de croire à une force magnétique qui guide avec assurance, et à des kilomètres de distance, l'abeille vers la ruche d'ou elle provient ". Elle se fait essentiellement en fonction de la position du soleil, mais d'après RIBBANDS (r953) les facteurs olfactifs pourraient jouer un rôle essentiel dans le phénomène du retour à la ruche.

LECOMTE (I956) a montré que lorsqu'on fait subir à une ruche une rotation de I $80^{\circ}$, il se forme une piste entre l'ancien emplacement du trou de vol et le nouveau. Les abeilles couvrent alors la distance entre ces deux points en marchant et non en volant. Cette piste peut persister pendant plus de 15 jours avant un retour à la nnrmale. 
Vuilithaume (I959) déplace une ruche de io mètres et la remplace par une ruche vide. Après plusieurs jours d'une importante perturbation les abeilles continuent à venir à l'ancien emplacement puis regagnent sans hésitation, en rasant le sol, le nouveau site de leur ruche. Leur nombre va en décroissant; cependant douze jours après la transposition certaines d'entre elles effectuent encore le parcours complexe. VUIILAUME démontre également, à la suite de BUTLER (I949) et de LEComte (1956) que la rétention mnémonique chez Apis mellifica peut atteindre et dépasser un mois.

Ces travaux et de nombreux autres, dont nous ne ferons pas la revue complète, démontrent la grande précision du sens de l'orientation chez l'Abeille. Cette précision n'est cependant pas absolue comme le prouve le phénomène appelé " dérive " par les apiculteurs. La dérive est une erreur d'orientation qui fait entrer, ou tenter d'entrer, 1'abeille dans une autre ruche que la sienne. L'observation courante a montré qu'elle peut être provoquée par différents facteurs :

a) présence d'un grand nombre de ruches de même taille et de même couleur, disposées en ligne droite sur un ou plusieurs rangs, orientées dans la même direction, très proches les unes des autres et à intervalles réguliers;

b) uniformité du site autour des ruches, sans point de repère permettant d'identifier telle ou telle zone du rucher;

c) miellée intense qui facilite l'acceptation des abeilles étrangères;

d) utilisation de ruchers couverts dont les colonies n'ont pas de signes distinctifs. Von FrIsch (I953) signale que I6 jeunes reines sur 2 I furent perdues, lors du vol de fécondation dans un tel rucher;

e) attraction des abeilles des ruches peu populeuses par les ruches voisines. Il est également probable que les ruches orphelines perdent un certain pourcentage de leur population au bénéfice de ruches pourvues d'une reine féconde.

La dérive peut s'orienter différemment en fonction de plusieurs facteurs :

a) Position relative des sources de nectar. Lorsque les ressources mellifères d'un rucher n'entourent pas ce rucher mais sont situées dans une direction privilégiée, la dérive se produit au profit des ruches qui se trouvent les plus près lors du retour des butineuses.

b) Présence d'obstacles naturels. - Les abeilles évitent tous les obstacles ou les masses sombres et passent par les brèches qui peuvent se présenter dans la végétation ou les constructions. Ce sont les chemins d'abeilles (LECOMTE, I956). Les ruches situées sur leur trajet bénéficient de la dérive.

c) Effet de position des ruches. - Les ruches situées dans les angles du rucher, aux extrémités des rangs, ou au premier rang, sont avantagées par la dérive lorsque les facteurs précédents sont faibles ou absents.

Le phénomène de dérive est facilement observable. En marquant des abeilles dans tune ruche on peut observer la présence d'un certain nombre d'individus marqués dans les ruches voisines au bout de quelques jours. Par ailleurs lorsque des colonies de races différentes sont voisines, on constate que leurs populations deviennent hétérogènes.

La dérive peut présenter de graves inconvénients pour l'expérimentation, la sélection ou 1'apiculture pratique. En effet :

a) C'est un facteur de propagation de toutes les maladies de l'abeille.

b) Elle fausse le rendement en miel des colonies et provoque donc des erreurs 
importantes en matière de sélection et en général dans toutes les expérimentations sur le terrain.

c) Elle provoque des erreurs lorsqu'on procède à des études biométriques pour caractériser les races ou les familles.

Le présent travail a pour but d'apporter quelques précisions chiffrées à nos connaissances actuelles qui sont uniquement basées sur l'observation.

Nous nous sommes particulièrement préoccupés de rechercher quelle est l'influence de la dérive sur le rendement en miel des ruchers. Par ailleurs nous avons étudié aussi précisément que possible les conditions qui favorisent l'apparition de la dérive.

\section{MATÉRIEL, ET MÉTHODE}

Pour réaliser notre expérience nous avons utilisé i 50 ruches du type Dadant Blatt io cadres modèle pastoral. Leur revêtement est constitué de peinture à l'huile à pigment d'aluminium. Ces ruches sont donc toutes semblables tant par la forme que par la couleur. L'essai a eu lieu pendant la floraison de la lavande et du lavandin dans la région du mont Ventoux. Les ruches de l'expérience ont été réparties dans trois ruchers :

Rucher I : destiné à subir une intense dérive;

Rucher II : destiné à subir une dérive moyenne;

Rucher III : destiné à subir une faible dérive.

Le rendement en miel des ruches ne correspond pas seulement à la quantité de miel prélevée dans la hausse, système simpliste peu conforme à la réalité, mais calculé à l'aide de la formule généralement utilisée pour nos essais.

dans laquelle :

$$
\mathrm{MH}+\mathrm{MC}+\left(\mathrm{P}_{1}-\mathrm{P}_{2}\right)-\mathrm{N}=\mathrm{R}
$$

MH $=$ Miel extrait de la (des) hausse ;

$\mathrm{MC}=$ Miel extrait des cadres de corps (s'il y a lieu);

$\mathrm{P}_{\mathbf{1}}=$ Poids de la ruche avant l'expérience ;

$\mathrm{P}_{\mathbf{2}}=$ Poids de la ruche après l'expérience ;

$\mathrm{N}=$ Nourrissement (s il y a lieu).

Le poids du miel récolté par les ruches est variable, même lorsqu'il n'y a pas de dérive. L'un des facteurs les plus importants de cette variabilité est le potentiel de ponte des reines quise traduit d'abord par une surface de couvain, puis par une population adulte plus ou moins importante. Nous avons mesuré les surfaces de couvain de toutes les ruches en expérience 20 jours avant la période de grande miellée (prévision approximative d'après l'état de plantes et les cycles annuels précédents). Les résultats sont domnés en " points " directement fournis par notre appareil à mesurer les surfaces de couvain (FRESNAYE, I962) et qui correspondent à $40 \mathrm{~cm}^{2}$ chacun ou ${ }_{5} 50$ cellules d'un rayon de couvain construit en cellules d'ouvrières. Le calcul des surfaces de couvain permet d'expliquer certains résultats qui autrement paraîtraient aberrants.

L'installation des ruches aux divers emplacements a eu lieu du 6 au 12 juin 1962.

Les surfaces de couvain ont été mesurées du 25 aul 27 juin.

La période de miellée importante se situe du i 5 au 30 juillet.

La récolte de miel fut effectuée du 2 I août au 30 août 1962.

Les surfaces de couvain étant toujours très faibles après la iniellée de la lavande et du lavandin, leur mesure ne s'imposait pas en fin d'expérience.

\section{RÉSULTATS}

\section{A. - Rucher I}

Le rucher I est installé au milieu d'une lavanderaie dont les rangs sont espacés d'environ I mètre. Tous les pieds de lavande sont du même âge et exactement semblables. Il n'existe aucun arbre, aucune autre plante, aucun rocher, rien qui 
puisse distinguer une partie du rucher d'une autre partie. L'extrême uniformité du site de ce rucher jointe à la totale similitude des ruches permet d'escompter un maximum de dérive.

Les ruches sont disposées sur deux rangs, distants d'environ Io mètres. Dans chaque rang les ruches sont espacées de $0,60 \mathrm{~m}$ à $0,70 \mathrm{~m}$ (fig. I) et ont toutes la même orientation. Pour distinguer chaque rang nous avons nommé " rang avant " celui qui est le plus proche quand on est placé face aux ruches, côté trou de vol, "rang arrière » celui qui est le plus éloigné.

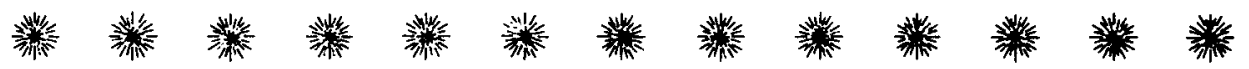 \begin{tabular}{|c|c|c|c|}
\hline$G$ & $E$ & $C$ & $A$ \\
\hline 潫
\end{tabular}

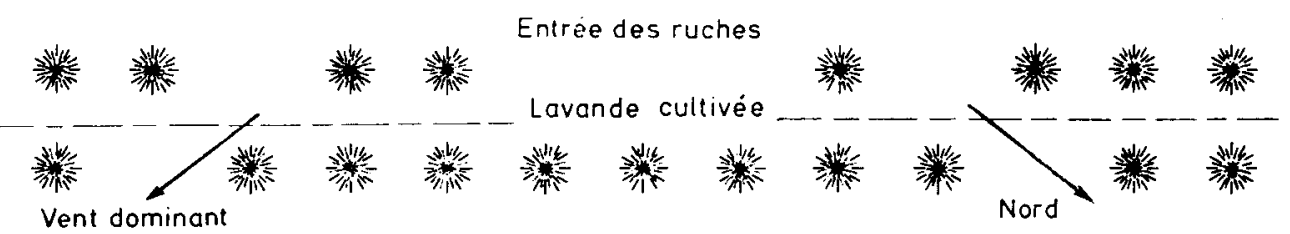

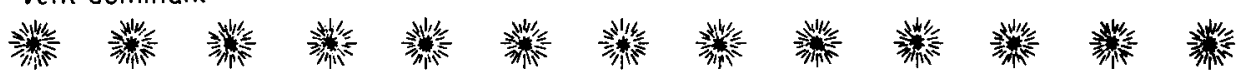

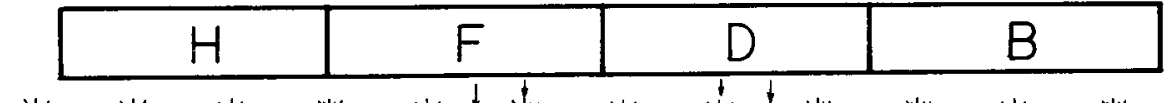

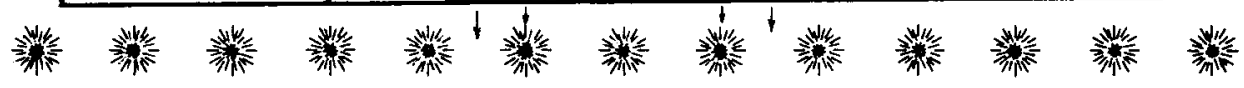 \\ FIG. I. - Rucher I. : Répartition des ruches entre les rangs d'un champ de lanande. Diaision du rucher en 8 lots A. B. C. D. E. F. G. H.}

Les histogrammes (fig. 2 et 3 ) représentent les rangs " avant » et "arrière " du rucher I. En abscisses les ruches sont disposées dans le même ordre que celui qu'elles occupaient sur le terrain dans chacun des deux rangs. Le numéro matricule de chaque ruche figure, sous la ligne de base, dans la colonne correspondante. En ordonnées, au dessus de la ligne de base, le rendement en miel de chaque ruche est indiqué en kilogrammes. Au dessous de la ligne de base les surfaces de couvain sont indiquées en points. Les ruches qui sont mortes au cours de l'expérience ont leur place indiquée; il en sera question plus loin.

Dans le rang avant on peut voir dès l'abord que la dérive a été énorme. Les huit ruches situées au centre du rang n'ont pas récolté de miel du tout ; puis en s'approchant des extrémités, la récolte augmente rapidement pour atteindre $5 \mathrm{I}, 200 \mathrm{~kg}$ dans le cas extrême de la ruche $\mathrm{n}^{\circ} 384$, et $4 \mathrm{I}, 300 \mathrm{~kg}$ pour la ruche $\mathrm{n}^{\circ} 426$. Outre leur position très favorable dans le rucher, ces deux ruches étaient en possession des plus importantes surfaces de couvain de tout le rucher. La réunion de ces deux facteurs explique la très nette culminance du rendement de ces colonies.

Dans le rang arrière le nombre de ruches n'ayant fait aucune récolte est beaucoup moins important, mais la dérive est cependant très forte. Les récoltes de miel 


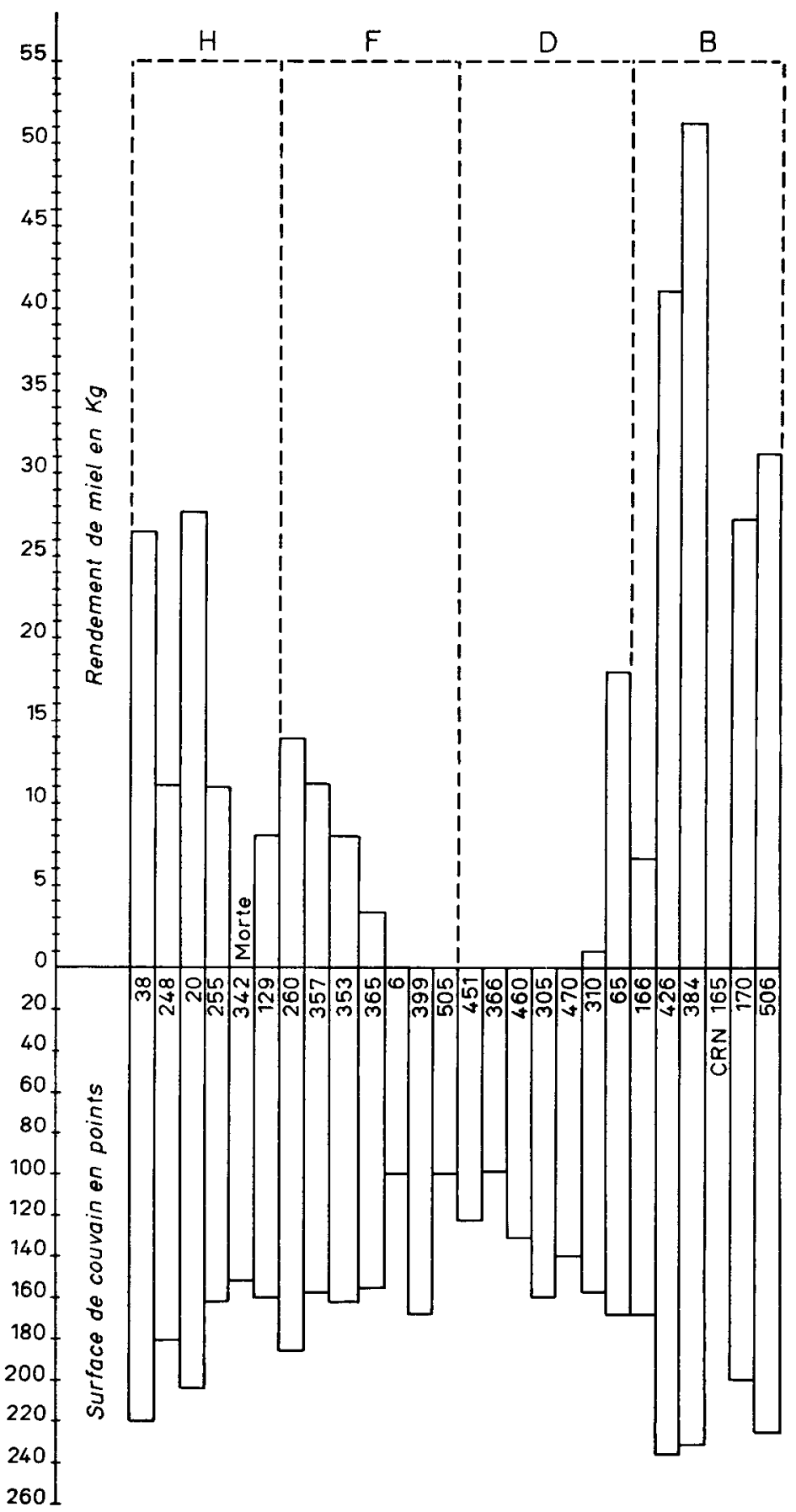

FIG. 2. - Rucher I : Rang avant. En abscisses, les ruches cont disposées dans le méme orlve que dans le rucher. En ordonnées, rendement en kilos de miel et surjace de couvain de chaque ruche. $C . R . N .=$ Cellules royales naturelles. 


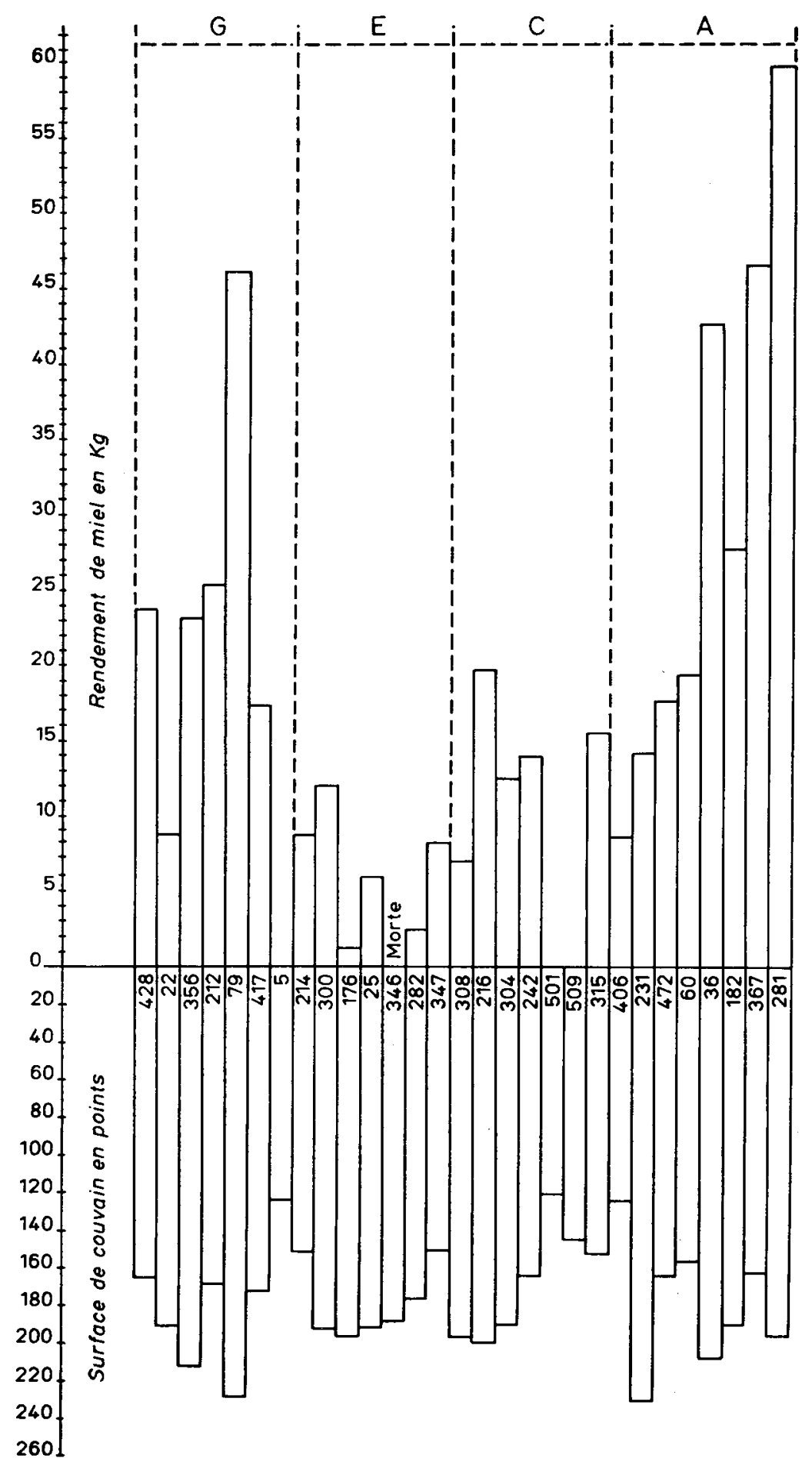

FIG. 3 -. Rucher I : Ranc arrière. En abscisses, les ruches sont disposées dans le même ordre que dans le rucher. En ordonnies, rendement en kilos de miel et surface de couvain de rhaque ruche 
sont dans l'ensemble plus importantes que dans le rang avant. La dérive s'est effectuée en outre du rang avant vers le rang arrière.

Dans l'ensemble du rucher, Io ruches ont un rendement absolument nul, soit près de $20 \mathrm{p}$. Ioo des effectifs. La moyenne générale du rucher, I5, I50 kg est fortement influencée par les mauvais résultats de ces ruches. Le meilleur rendement est celui de la ruche $\mathbf{n}^{0} 28 \mathrm{I}$, qui atteint $58,900 \mathrm{~kg}$.

TABLEAU I

\begin{tabular}{|c|c|c|c|c|c|}
\hline \multicolumn{6}{|c|}{ Rucher I } \\
\hline No Ruche & $\begin{array}{c}\text { Rendement } \\
(\mathrm{kg})\end{array}$ & & No Ruche & $\begin{array}{l}\text { Rendement } \\
(\mathrm{kg})\end{array}$ & \\
\hline $\begin{array}{r}281 \\
367 \\
182 \\
36 \\
60 \\
472 \\
231 \\
406\end{array}$ & $\begin{array}{r}58,9 \\
46,5 \\
27,6 \\
42,9 \\
18,5 \\
17,7 \\
14,0 \\
8,8\end{array}$ & $\begin{array}{l}\text { A } \\
\text { Rdt total 234,9 } \mathrm{kg} \\
\text { Nbre de ruches } 8 \\
\text { Rdt moyen } 29,36 \mathrm{~kg}\end{array}$ & $\begin{array}{l}506 \\
170 \\
165 \\
384 \\
426 \\
166\end{array}$ & $\begin{array}{c}31,4 \\
27,3 \\
\text { morte } \\
51,2 \\
41,3 \\
6,7\end{array}$ & $\begin{array}{l}\text { B } \\
\text { Rdt total } 157,9 \mathrm{~kg} \\
\text { Nbre de ruches } 5 \\
\text { Rdt moyen } 31,58 \mathrm{~kg}\end{array}$ \\
\hline $\begin{array}{l}315 \\
509 \\
501 \\
242 \\
304 \\
216 \\
308\end{array}$ & $\begin{array}{c}15,9 \\
0 \\
0 \\
14,0 \\
12,5 \\
19,7 \\
7,5\end{array}$ & $\begin{array}{l}\text { C } \\
\text { Rdt total } 69,6 \mathrm{~kg} \\
\text { Nbre de ruches } 7 \\
\text { Rdt moyen } 9,94 \mathrm{~kg}\end{array}$ & $\begin{array}{r}65 \\
310 \\
470 \\
305 \\
460 \\
366 \\
451\end{array}$ & $\begin{array}{c}18,0 \\
1,0 \\
0 \\
0 \\
0 \\
0 \\
0\end{array}$ & $\begin{array}{l}\text { D } \\
\text { Rdt total } 19,0 \mathrm{~kg} \\
\text { Nbre de ruches } 7 \\
\text { Rdt moyen } 2,71 \mathrm{~kg}\end{array}$ \\
\hline $\begin{array}{r}347 \\
282 \\
346 \\
25 \\
176 \\
300 \\
214\end{array}$ & $\begin{array}{c}8,2 \\
2,5 \\
\text { morte } \\
5,8 \\
1,2 \\
12,2 \\
8,4\end{array}$ & $\begin{array}{l}\text { E } \\
\text { Rdt total } 38,3 \mathrm{~kg} \\
\text { Nbre de ruches } 6 \\
\text { Rdt moyen } 6,38 \mathrm{~kg}\end{array}$ & $\begin{array}{r}505 \\
399 \\
6 \\
365 \\
353 \\
357 \\
260\end{array}$ & $\begin{array}{r}0 \\
0 \\
0 \\
3,2 \\
8,0 \\
11,5 \\
13,9\end{array}$ & $\begin{array}{l}\text { F } \\
\text { Rdt total } 36,6 \mathrm{~kg} \\
\text { Nbre de ruches } 7 \\
\text { Rdt moyen } 5,22 \mathrm{~kg}\end{array}$ \\
\hline $\begin{array}{r}5 \\
417 \\
79 \\
212 \\
356 \\
22 \\
428\end{array}$ & $\begin{array}{r}2,0 \\
17,3 \\
46,3 \\
24,5 \\
23,4 \\
8,9 \\
23,9\end{array}$ & $\begin{array}{l}\text { G } \\
\text { Rdt total } 146,3 \mathrm{~kg} \\
\text { Nbre de ruches } 7 \\
\text { Rdt moyen } 20,90 \mathrm{~kg}\end{array}$ & $\begin{array}{r}129 \\
342 \\
255 \\
20 \\
248 \\
38\end{array}$ & $\begin{array}{c}8,0 \\
\text { morte } \\
11,0 \\
27,6 \\
12,3 \\
26,5\end{array}$ & $\begin{array}{l}\text { H } \\
\text { Rdt total } 85,4 \mathrm{~kg} \\
\text { Nbre de ruches } 5 \\
\text { Rdt moyen } 17,08 \mathrm{~kg}\end{array}$ \\
\hline & & $\begin{array}{l}\text { Rendement total du } \\
\text { Nombre de ruches } \\
\text { Rendement moyen }\end{array}$ & rucher & $\begin{array}{r}\mathrm{kg} \\
, 15 \mathrm{~kg}\end{array}$ & \\
\hline
\end{tabular}


Une étude des rendements moyens nous permet d'aller plus avant dans ces constatations. Pour cette étude nous avons divisé le rucher en 8 lots ainsi qu'il est indiqué fig. I, afin d'éliminer les variations inhérentes à chaque ruche prise isolément.

Nous indiquons dans le tableau I, pour chaque lot, le rendement de chaque ruche, le rendement total, le nombre de ruches et le rendement moyen en $\mathrm{kg}$ de miel.

Dans ce rucher, les lots $\mathrm{A}$ et $\mathrm{B}$ sont les grands bénéficiaires de la dérive. Les lots $\mathrm{G}$ et $\mathrm{H}$ viennent ensuite avec une moyenne un peu plus faible. Enfin les lots $\mathrm{C}$ et $\mathrm{E}$ sont légèrement supérieurs aux lots $\mathrm{D}$ et $\mathrm{F}$ qui sont les plus défavorisés. La dérive a donc dirigé les butineuses vers les extrémités des rangs du rucher.

\section{B. - Rucher II}

Le rucher II est installé dans un terrain inculte ou poussent de la lavande et quelques pieds de chêne vert (Quercus Ilex). Ce site, disposant de peu de points de repère, permet d'escompter une dérive moyenne, moins importante que dans le rucher I. Les ruches y sont également alignées sur deux rangs (fig. 4), orientées du même côté, L'écartement entre les colonies est le même qu'au rucher I. Les champs de lavande cultivée sont très proches et de même densité qu'au rucher I.

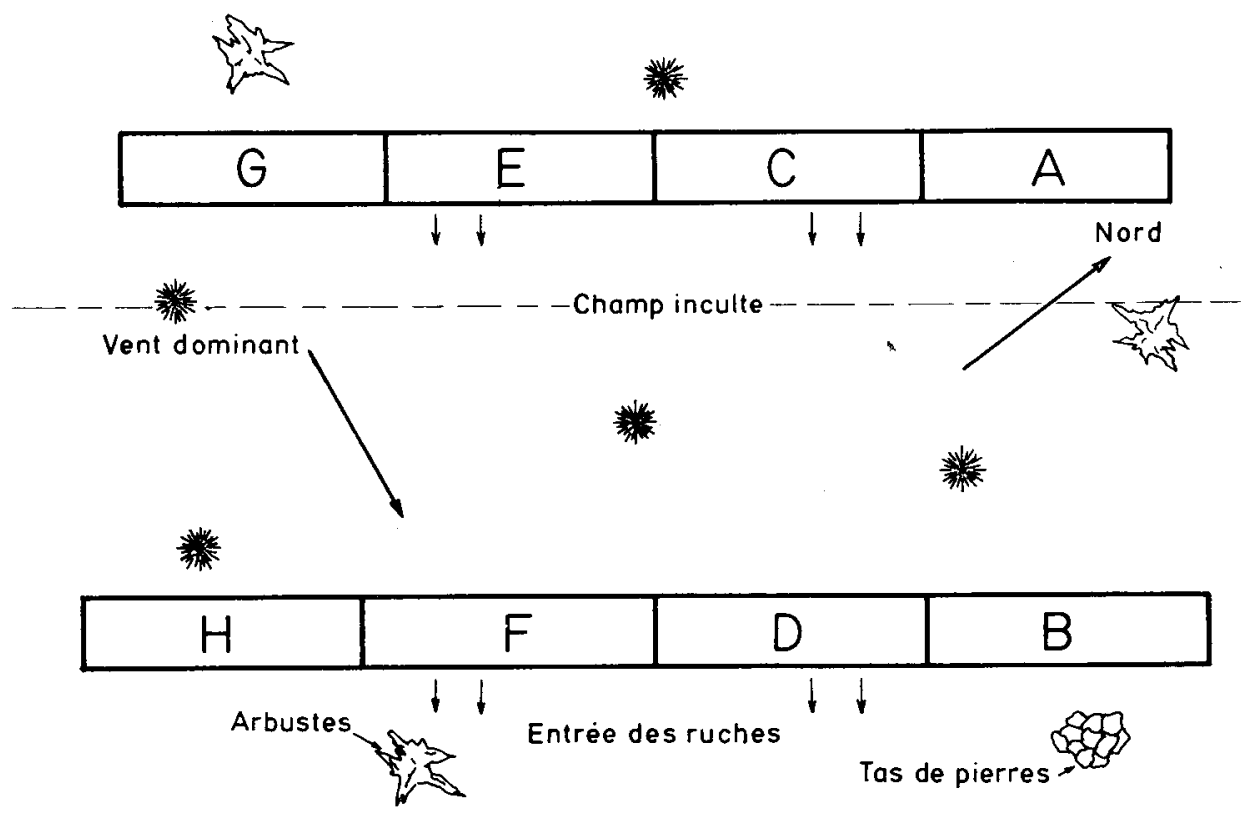

FIG. 4. - Rucher II : Repartition des ruches sur deux rangs dans un terrain inculte Division du mener en 8 lots A. B. C. D. E. F. G. H.

Les histogrammes (fig. 5 et 6) représentent les rangs " avant " et " arrière " de ce rucher. Ils correspondent aux mêmes normes que ceux du rucher I. Dans ce rucher les différences de récoltes dues à la dérive sont beaucoup moins nettes bien qu'encore visibles dans le rang arrière. Aucune ruche n'a fait de rendement nul. 


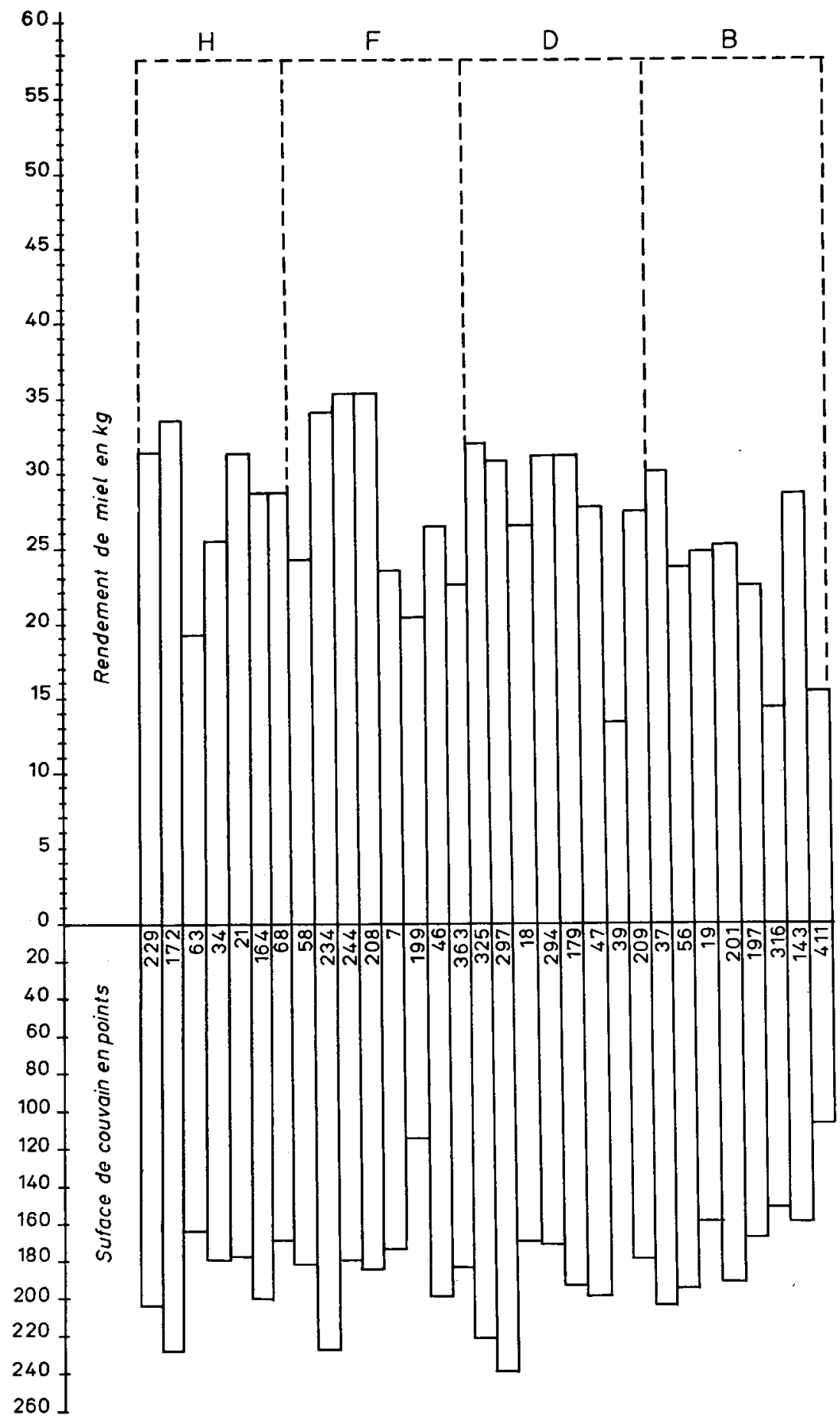

Frr. 5. - Rucher II : Rang avant. En abscisses, les ruches sont disposées dans le mème ordre que dans le rucher. En ordonnées, rendement en kilos de miel et surface de couvain de chaque ruche. 


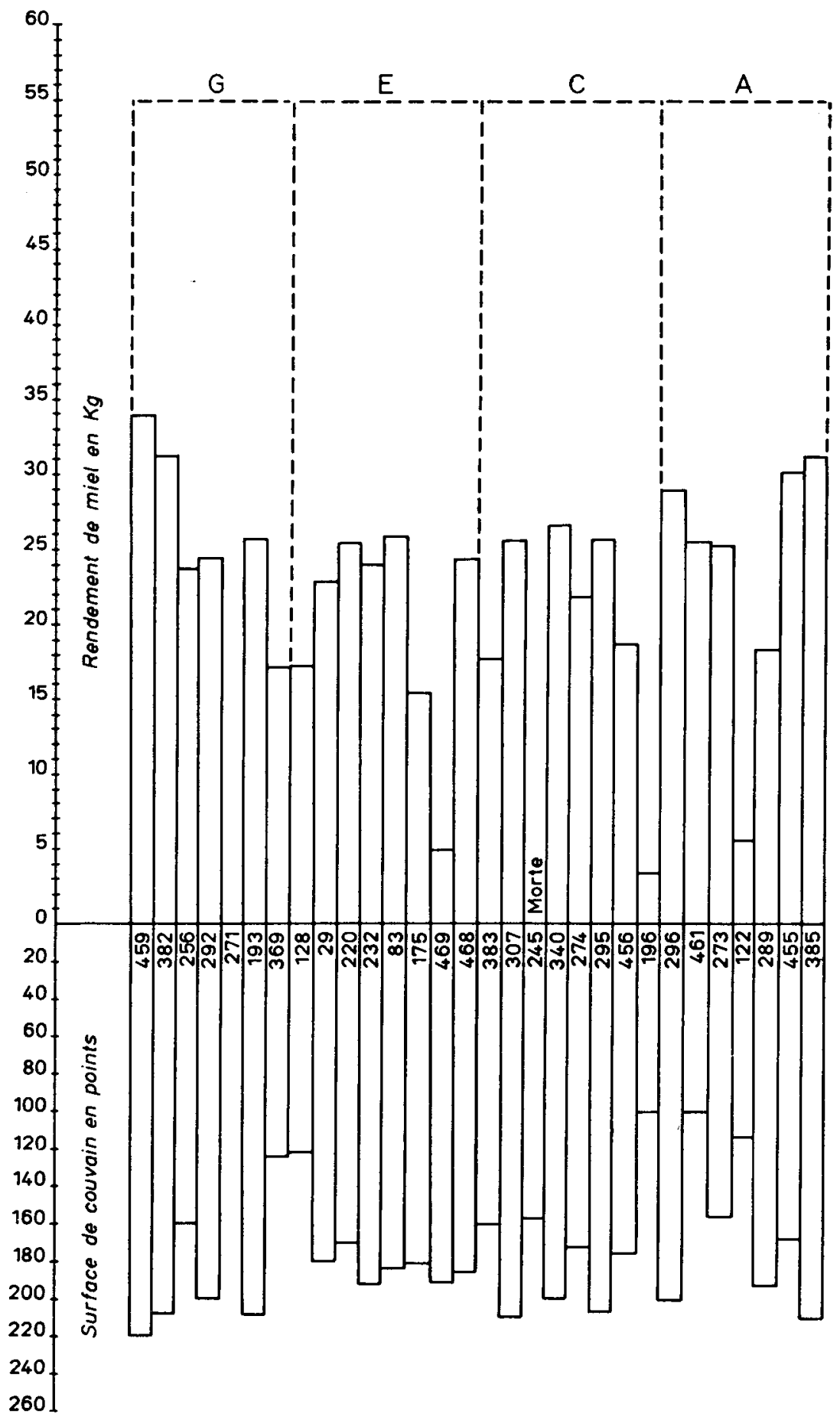

FIG. 6. - Rucher II : Rang arrière. En abscisses, les ruches sont disposées dans le méme ordre que dans le rucher. En ordonnées, rendement en kilos de miel et surface de couvain de chaque ruche 
Ici la dérive s'est surtout effectuée du rang arrière vers le rang avant et l'étude statistique nous permet de mieux la définir. Comme précédemment nous avons divisé ce rucher en 8 lots (fig. 4). Les lots D-F-H sont les plus favorisés. L,e lot B équivaut aux lots $\mathrm{A}$ et $\mathrm{G}$, les lots $\mathrm{C}$ et $\mathrm{E}$ ont des rendements plus faibles (tableau 2 ). La dérive d'arrière en avant s'accompagne d'une dérive vers les extrémités du rang arrière comme dans le rucher I.

\section{TABLEAU 2}

Rucher II

\begin{tabular}{|c|c|c|c|c|c|}
\hline No Ruche & $\begin{array}{c}\text { Rendement } \\
(\mathrm{kg})\end{array}$ & & No Ruche & $\begin{array}{l}\text { Rendement } \\
(\mathrm{kg})\end{array}$ & \\
\hline $\begin{array}{l}385 \\
455 \\
289 \\
122 \\
273 \\
461 \\
296\end{array}$ & $\begin{array}{r}31,9 \\
31,1 \\
18,3 \\
5,6 \\
27,5 \\
25,3 \\
28,6\end{array}$ & $\begin{array}{l}\text { Rdt total } 165,3 \mathrm{~kg} \\
\text { Nbre ruches } 7 \\
\text { Rdt moyen } 23,61 \mathrm{~kg}\end{array}$ & $\begin{array}{r}411 \\
143 \\
316 \\
197 \\
201 \\
19 \\
56 \\
37\end{array}$ & $\begin{array}{l}15,3 \\
28,1 \\
14,3 \\
22,5 \\
25,1 \\
24,7 \\
22,9 \\
30,2\end{array}$ & $\begin{array}{l}\text { Rdt total } 183,1 \mathrm{~kg} \\
\text { Nbre ruches } 8 \\
\text { Rdt moyen } 22,88 \mathrm{~kg}\end{array}$ \\
\hline $\begin{array}{l}196 \\
456 \\
295 \\
274 \\
340 \\
245 \\
307 \\
383\end{array}$ & $\begin{array}{c}3,4 \\
18,7 \\
26,8 \\
22,0 \\
26,9 \\
\text { morte } \\
25,7 \\
17,7\end{array}$ & $\begin{array}{l}\text { C } \\
\\
\text { Rdt total } 141,2 \quad \mathrm{~kg} \\
\text { Nbre ruches } 7 \\
\text { Rdt moyen } 20,17 \mathrm{~kg}\end{array}$ & $\begin{array}{r}209 \\
39 \\
47 \\
179 \\
294 \\
18 \\
297 \\
325\end{array}$ & $\begin{array}{l}27,1 \\
13,5 \\
27,5 \\
31,1 \\
31,1 \\
26,5 \\
30,9 \\
32,0\end{array}$ & $\begin{array}{l}\text { Rdt total } 220,0 \mathrm{~kg} \\
\text { Nbre ruches } 8 \\
\text { Rdt moyen } 27,50 \mathrm{~kg}\end{array}$ \\
\hline $\begin{array}{r}468 \\
469 \\
175 \\
83 \\
232 \\
220 \\
29 \\
128\end{array}$ & $\begin{array}{r}24,5 \\
5,0 \\
15,5 \\
26,0 \\
24,0 \\
25,5 \\
23,0 \\
17,0\end{array}$ & $\begin{array}{l}\text { F } \\
\\
\text { Rdt total } 160,5 \quad \mathrm{~kg} \\
\text { Nbre ruches } 8 \\
\text { Rdt moyen } 20,06 \mathrm{~kg}\end{array}$ & $\begin{array}{r}363 \\
46 \\
199 \\
7 \\
208 \\
244 \\
234 \\
58\end{array}$ & $\begin{array}{l}22,6 \\
26,3 \\
20,3 \\
23,2 \\
31,2 \\
35,1 \\
34,2 \\
24,1\end{array}$ & $\begin{array}{l}\text { Rdt total } 217,0 \mathrm{~kg} \\
\text { Nbre ruches } 8 \\
\text { Rdt moyen } 27,12 \mathrm{~kg}\end{array}$ \\
\hline $\begin{array}{l}369 \\
193 \\
271 \\
292 \\
256 \\
382 \\
459\end{array}$ & $\begin{array}{c}17,1 \\
25,5 \\
0 \\
24,5 \\
23,8 \\
31,3 \\
34,0\end{array}$ & $\begin{array}{l}\text { Rdt total } 156,2 \mathrm{~kg} \\
\text { Nbre ruches } 7 \\
\text { Rdt moyen } 20,31 \mathrm{~kg}\end{array}$ & $\begin{array}{r}68 \\
16 ! \\
21 \\
31 \\
63 \\
172 \\
229\end{array}$ & $\begin{array}{l}28,8 \\
28,5 \\
31,3 \\
25,4 \\
18,1 \\
33,5 \\
31,1\end{array}$ & $\begin{array}{l}\text { Rdt total } 196,7 \mathrm{~kg} \\
\text { Xbre ruches } 7 \\
\text { Rdt moyen } 28,10 \mathrm{~kg}\end{array}$ \\
\hline
\end{tabular}

Rendement total des ruches

Nombre de ruches

Rendement moyen
$1440 \mathrm{~kg}$ 


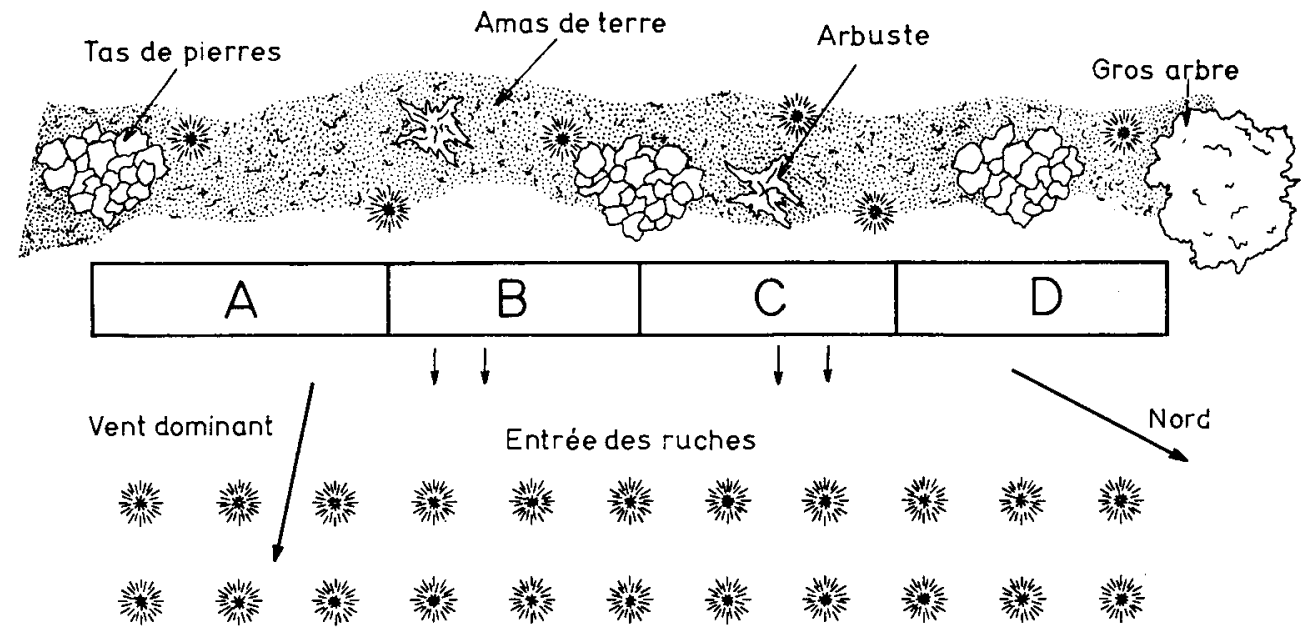

Lavande cultivée

FIG. 7. - Rucher III : Répartition des ruches sur un rang, le long d'une haie variée. Division du rucher en 4 lots $A . B . C . D$.

TABLEAU 3

Rucher III

\begin{tabular}{|c|c|c|c|c|c|}
\hline No Ruche & $\begin{array}{l}\text { Rendement } \\
(\mathrm{kg})\end{array}$ & & No Ruche & $\begin{array}{l}\text { Rendement } \\
(\mathrm{kg})\end{array}$ & \\
\hline & & \multirow{10}{*}{$\begin{array}{l}\text { Rdt total } 228,7 \mathrm{~kg} \\
\text { Nbre ruches } 9 \\
\text { Rdt moyen } 25,4 \mathrm{~kg}\end{array}$} & & & \multirow{10}{*}{$\begin{array}{l}\text { Rdt total } 263,2 \mathrm{~kg} \\
\text { Nbre ruches } 8 \\
\text { Rdt moyen } 32,9 \mathrm{~kg}\end{array}$} \\
\hline 269 & 12 & & 410 & 25,2 & \\
\hline 109 & 40,3 & & 102 & 32,4 & \\
\hline 113 & 28,8 & & 387 & 32,3 & \\
\hline 276 & 9,2 & & 512 & 26,8 & \\
\hline 547 & 32,2 & & 71 & 32,4 & \\
\hline 486 & 24,5 & & 349 & 32,8 & \\
\hline 265 & 43,8 & & 480 & 33,0 & \\
\hline 481 & 29,2 & & 474 & 48,3 & \\
\hline 437 & 8,7 & & & & \\
\hline & & \multirow{9}{*}{$\begin{array}{l}\text { Rdt total } 223,8 \mathrm{~kg} \\
\text { Nbre ruches } 8 \\
\text { Rdt moyen } 27,9 \mathrm{~kg}\end{array}$} & & & \multirow{9}{*}{$\begin{array}{l}\text { Rdt total } 238,0 \mathrm{~kg} \\
\text { Nbre ruches } 8 \\
\text { Rdt moyen } 29,7 \mathrm{~kg}\end{array}$} \\
\hline 252 & 32,4 & & 550 & 37,2 & \\
\hline 523 & 18,0 & & 519 & 27,7 & \\
\hline 518 & $1 / 4, t^{\prime}$ & & 521 & 11,2 & \\
\hline 50 & 27,3 & & 515 & 21,6 & \\
\hline 80 & 31,7 & & 516 & 42,6 & \\
\hline 520 & 13,7 & & 522 & 13,3 & \\
\hline 73 & 40,8 & & 277 & 26,4 & \\
\hline 514 & 35,5 & & 131 & 28,0 & \\
\hline
\end{tabular}

Rendement total du rucher

Nombre de ruches

Rendement moyen
$953,7 \mathrm{~kg}$ 


\section{C. - Rucher III}

'L r rucher III est installé en bordure d'un champ de lavande cultivée dont les premiers rangs se trouvent à 3 mètres des ruches. Les ruches sont alignées, sur un seul rang, et à égale distance les unes des autres (comme aux ruchers I et II) devant une haie, qui est constituée par des rochers, différents arbustes, et quelques arbres (fig. 7). La diversité du site est donc suffisante pour permettre aux abeilles de se repérer facilemént. Seule la monotonie de teinte, de forme et d'alignement des ruches peut causer dies perturbations de repérage.

L'histogramme de ce rucher (fig. 8) montre surtout la grande diversité de rendement entre les ruches quelle que soit leur position.

Ce rucher, composé de 33 colonies seulement, est divisé en quatre lots (tableau 3). La variation du rendement moyen entre ces lots est assez peu importante. Par contre la variation à l'intérieur des lots est très forte et montre l'hétérogénéité des colonies. Toutes les ruches ont récolté du miel.

Ainsi ep ldépit de l'alignement régulier et de la similitude des ruches, aucune dérive notable n'est à signaler dans ce rucher. Les repères du site qui entoure le rucher ont suffi à l'orientation des butineuses. Il n'est donc pas nécessaire de peindre les ruches de couleurs différentes dans les ruchers modernes lorsqu'ils sont placés dans des sites compqrtant des repères variés.

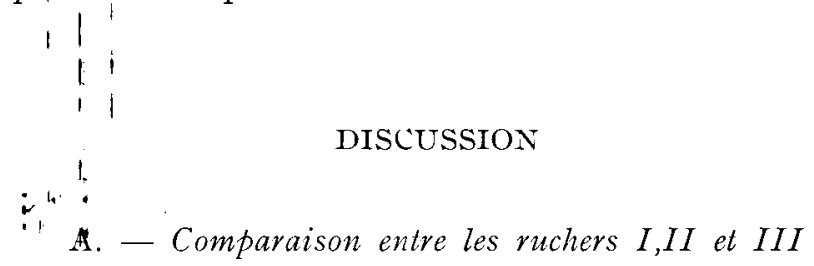

L'analyse des rendements en miel obtenus dans les trois rûchers de nos essais doit être complétée par la comparaison de ces ruchers entre eux.

I es ressources mellifères des trois emplacements sont similaires et se composent essenti`llement de lavande et de lavandin cultivés sur de grandes étendues à l'exclusion de toute autre culture. Le rucher I et le rucher II sont distants d'environ 700 mètres alors que le rucher III est situé à $2 \mathrm{~km}$ des deux premiers.

Pour chacun des trois ruchers la moyenne générale du rendement est la suivante :

$$
\begin{aligned}
& \text { Rucher I }=25, \text { I5 } \mathrm{kg} \\
& \text { Rucher II }=24 \mathrm{~kg} \\
& \text { Rucher III }=28,90 \mathrm{~kg}
\end{aligned}
$$

Compte tenu de la faible distance qui sépare les ruchers I et II, de la similitude de la flore et de sa densité, il semble que l'importante différence de la moyenne générale entre ces deux ruchers $(8,85 \mathrm{~kg})$ soit due en majeure partie à 1'importante dérive qui s'est produite au rucher I. Théoriquement, le nombre total d'abeilles du rucher ne devrait pas avoir sensiblement varié, puisque les butineuses qui ont dérivé sont venues renforcer les colonies voisines. On peut donc se demander quelles sont les raisons de la réduction du rendement moyen lorsqu'il y a dérive. Voici quelques éléments de réponse à cette question : 


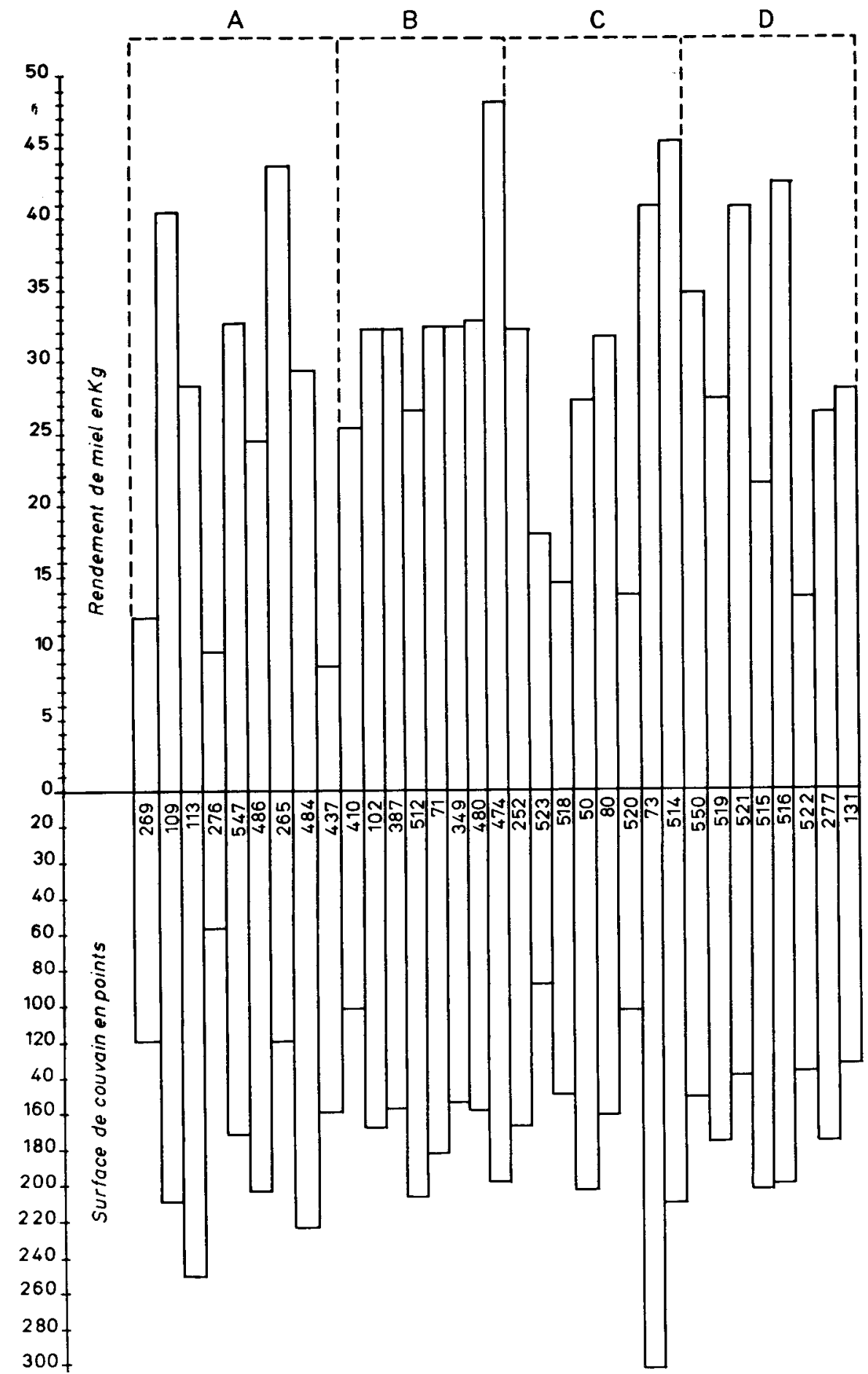

FIG. 8. - Rucher III : En abscisses, les ruches sont disposées dans le méme ordre que dans le rucher. En ordonnées, rendement en kilos de miel et surjace de courain de chaque ruche. 
a) Les colonies défavorisées par la dérive accusent beaucoup plus de déficit que les colonies favorisées n'enregistrent de bénéfice ;

b) Parmi les abeilles qui dérivent, certaines ne sont pas acceptées par les ruches voisines et sont mises à mort. En outre, les erreurs de repérage imposent aux colonies une augmentation du nombre de gardiennes à l'entrée.

c) Les abeilles qui dérivent ne reconnaissent pas l'odeur spécifique de leur colonie et perdent beaucoup de temps avant d'entrer dans une ruche.

d) Une dérive intense déclenche sans aucun doute un " pillage " qui nuit à la récolte. Dans certains cas le pillage peut aller jusqu'à la mise à mort des colonies attaquées. C'est ainsi que dans le rucher I trois colonies sont mortes pendant l'expérience, alors qu'une seule est morte dans le rucher II à moindre dérive et aucune dans le rucher III. Il semble que l'augmentation du nombre de colonies détruites à la suite du pillage puisse être due à la forte dérive qui sévissait au rucher I.

Dans certains cas extrêmes, la dérive affaiblissant au maximum certaines colonies, l'élevage peut s'en trouver affecté. L'histogramme du rang avant du rucher I montre que les colonies du centre, qui n'ont fait aucune récolte possèdent, zo jours après leur installation, une surface de couvain inférieure en moyenne à celle des colonies plus proches des extrémités du rang. Le déséquilibre entre les abeilles " d'intérieur " et les abeilles " d'extérieur ", provoqué par la perte continuelle de ces dernières, oblige les jeunes abeilles à devenir très tôt des butineuses (qui dérivent à leur tour) réduisant ainsi le nombre de " nourrices " qui assurent l'élevage du couvain. Par voie de conséquence celui-ci se restreint peu à peu donnant naissance à moins d'abeilles. Ainsi la colonie s'affaiblit progressivement sans possibilité de se renforcer.

Le vent, qui a cependant été fréquent et fort pendant l'expérience, n'a pas eu d'influence sur la dérive. In effet, celle-ci s'est effectuée contre le vent dans le rucher $I$ et dans le sens du vent au rucher $I I$.

I a pente du terrain dans les ruchers semble être un élément de dérive, car dans le rucher $I$ le rang arrière qui se trouve plus bas que le rang avant est bénéficiaire de la dérive. Il est difficile de savoir si cela est dû à la pente du terrain ou à la direction de la plus forte miellée. Cependant on peut identifier cette dérive à la répugnance qu'ont les abeilles à survoler les obstacles. Ainsi les zones basses d'un rucher seraient les plus favorisées.

\section{$\mathrm{B}$ - Méthodes de lutte contre la dérive}

Nous avons montré que les inconvénients de la dérive sont divers et peuvent parfois être très graves pour les colonies. Il y a donc tout intérêt à lutter le plus possible contre elle dans les ruchers modernes. Pour cela on utilisera les méthodes suivantes :

$\left.I^{0}\right)$ Installer les ruchers dans des sites comportant le plus possible de repères discernables par les abeilles, arbres, arbustes, rochers, constructions.

$\left.2^{\circ}\right)$ Éviter de placer un trop grand nombre de ruches au même emplacement. Soixante ruches constituent un maximum qu'il serait préférable de ne pas dépasser ; trente à quarante ruches un nombre optimum.

$\left.3^{\circ}\right)$ Accentuer la diversité du site en disposant les ruches irrégulièrement; donner aux ruches des orientations différentes; varier la distance qui les sépare; laisser pousser des arbustes ou des plantes entre les groupes de ruches, faire varier 
la distance entre les ruches et le sol. Essayer surtout " d'utiliser le terrain " au maximum.

$\left.4^{\circ}\right)$ Lorsque le site des ruchers ne se prête pas à ces dispositions on peut égaement peindre les ruches de couleurs différentes : blanc, jaune, bleu et noir.

Reçu pour publication en avril 1963 .

\section{SUMMARY}

ORIENTATION ERRORS OF BEES (DRIFT) IN THE MODERN APIARY

Errors in the orientation of bees, known in apiculture as "drift " are due to uniformity of the site in which the hives are situated. This monotonous effect is strengthened by the identical nature of the hives, both in shape and colour.

Disorientation can attain considerable proportions when the site of the apiary is absolutely uniform. In such a site there may be no yield from $20 \mathrm{p}$. 100 of the hives, whilst the mean yield is $15 \mathrm{~kg}$, and certain hives can produce up to $60 \mathrm{~kg}$ of honey.

The disorientation causes a lowering of the average yield of honey in certain apiaries, sometimes to a marked degree, and a reduction in the incubation area of colonies, with consequent weakening of the latter.

It appears that acute disorientation can trigger off hiverobbing which leads to destruction of the attacked colonies in extreme cases.

\section{RÉFÉRENCES BIBLIOGRAPHIQUES}

Butler C. G., I 949 . Bee Behaviour. Nature, 163, I $20-200$.

FREE J. B. 1958. The drifting of honey bees. J. agric. Sci., 51 (3), 294-306.

Fresnaye J., 1962. Un appareil pour le calcul rapide des surfaces de couvain dans les ruches. Ann. Abeille, $5,145^{-1} 53$.

Von Friscir K., I953. Aus dem Leben der Bienen, 5, Springer-Verlag. Berlin.

Von Frisch K., Lindauer M., I954. Himmel und Erde in Konkurrenz bei der Orientierung der Bienen. Naturviss. 2, 245-253.

LeComte J,, I956. Formation de pistes par les butineuses d'une ruche ayant subi une rotation de $180^{\circ}$. Zeit. fïr Bien, April, I-4.

Lecomte J., 1956. Sur le vol des abeilles butineuses. Zeit. für. Tierpsych. 1, 26-30.

RibBands C. R., 1953. The adaptability of the Homecoming. Honeybee. J. An. Behav. 1, 59-66.

Vuillaume M., I959. La retention mnémonique chez Apis mellifica. Ann. Abeille, 2, i59-170. 